\title{
Gradient Coils Design with Regularization Method for Superconducting Magnetic Resonance Imaging
}

\author{
S. Zheng*, X. Li *, W. Yan ${ }^{\dagger}$, W. Xu*, and X. Liu* \\ * China Jialiang University, Hangzou China \\ $\dagger$ Auckland University of Technology, Auckland, New Zealand
}

\begin{abstract}
This paper proposes an approach to the design of gradient coils for superconducting magnetic resonance imaging (MRI). The designed method takes use of Fourier series expansions to describe the continuous current density of the coil surface and then employs stream function method to extract the coil patterns. During the numerical simulation, a linear equation is constructed and solved using a Tikhonov regularization scheme. Using this method, the gradient coils with high level of linearity are designed. Our contributions in this paper are to expend the current densities of coils into Fourier series analytically as well as optimize the parameters of regularization from the plotted curve.
\end{abstract}

Index Terms-superconducting magnetic resonance imaging, stream function, gradient coil, Tikhonov regularization

\section{INTRODUCTION}

Gradient coils are important components of magnetic resonance imaging (MRI) which have been used to get the information of spatial location of the sample being imaged [1]. Generally, there are three sets of gradient coils to be designed so as to generate gradient magnetic fields which are parallel to the magnet's main field and vary linearly in an imaging space (usually a diameter of spherical volume, DSV) along the three axes of a rectangular coordinate system. Assume the direction of the main magnetic field is aligned with $z$ axis, we will focus on the design of gradient coils where the $z$ component of the magnetic flux density, $B_{z}$, vary linearly along the $x, y$ and $z$ axes of the DSV.

Image quality is directly dependent on the performance of the employed gradient coils. In designing the gradient coils, the linearity and eddy currents must be taken into consideration:

- Linearity. The linearity of the gradient magnetic field has a direct effect on the image resolution. The gradient coils with poor linearity will result in serious image distortion. Generally, the nonlinearity of the gradient magnetic field is required not to be higher than 5\%.

- Eddy currents. When gradients are switched rapidly, eddy currents are induced in conductors surrounding outside the gradient coils, which will cause additional magnetic fields in the DSV and worsen the image quality. The application of active shielding coils, which consist of primary coil and shielding coil, is an effective way to get desired gradient fields in the DSV and reduce the magnetic fields nearly to zero outside the coils [2]. Generally, the magnetic flux density in the shielded region, generated by primary and shielding coils, is not in excess of 5 Gauss ( $1 \mathrm{~T}=10^{4}$ Gauss).

A classical design method for gradient coils is target field method proposed by Turner [3]-[5]. It is one of the current density distribution method. The size of the coil can not be specified in advance with this method, the Fourier transform is used with a constraint added and the current density must vanish outside a specified finite area [6]-[9]. In some cases, smoothing functions have to be incorporated in the Fourier transform to guarantee its convergence, which will introduce unnecessary errors and complications. In order to solve this problem, many researchers gave an effective design methodology of the finite-size gradient coils for superconducting MRI system [10] [11] and permanent magnet MRI system [12] [13]. Only several sample points in the DSV are adopted, a function is constructed in terms of stored energy, the $z$ component of magnetic fields and the constrained current density, so there is high nonlinearity in their designs.

In previous work, a penalty function was often used to solve ill-conditioned problem of the equations. Different choices for the parameter norm can result in different solutions being preferred. For the optimization design of discrete wires gradient coil, to find the highest gradient field or linearity of the gradient coil with particular number of turns, a norm of the magnetic fields in the shielded region is added [14] [15]. The constraint of stored energy or dissipated power is not easily added in this method. One of the current density method with Tikhonov regularization was applied in [16], but the selection of regularization parameter was not given in detail.

Our contributions in this paper are to expend the current densities of both primary and shielding coils into Fourier series analytically in given finite-size coils former as well as optimize the parameters of regularization from the plotted curve.

In this paper, in Section II we will introduce the related work, our research method will be detailed in Section III, our experimental results will be demonstrated in Section IV and our conclusion will be drawn in Section V, we also will vision our future work in the section.

\section{RELATED WORK}

In this paper, a finite-size gradient coils design with Tikhonov regularization method for superconducting MRI system is presented. Gradient coils are mounted on the cylindrical forms, the current densities of coils are expanded into Fourier series in a cylindrical coordinate system; the Fourier 


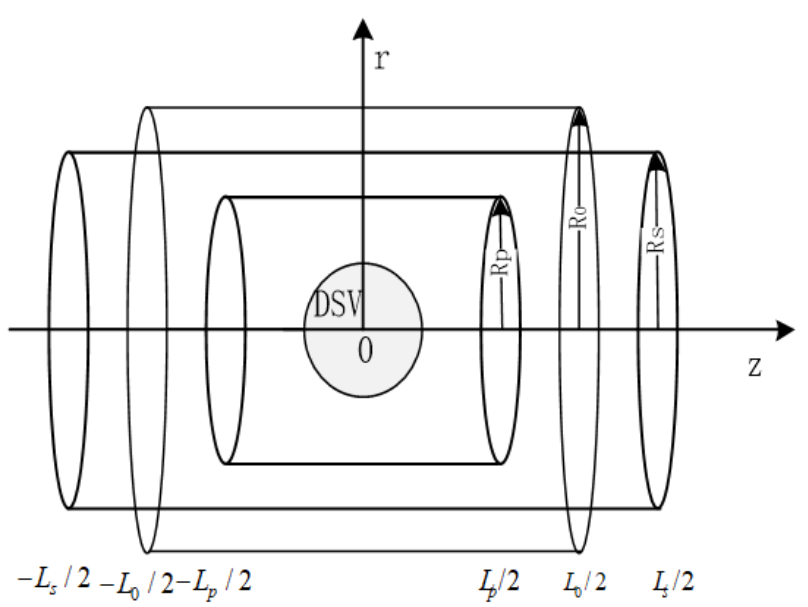

Fig. 1. The schematic diagram of gradient coils for superconducting MRI system

coefficients are variables to be solved. A large number of sample points are selected in the DSV and the shielded region, a cost function that relates the computed field to the desired field is constructed and a Tikhonov regularization function is added to solve the ill-condition problem. When the current densities are obtained, a stream function is used to discretize the current densities [17]. Once this discretization process is completed, the magnetic fields, yielded from these currentcarrying wires, are calculated using the Biot-Savart law.

A popular Tikhonov regularization method was L-curve presented by Hansen [18]. In this plot, the log-log scale was used, the norm of the solution on the ordinate against the norm of the residual on the abscissa, and regularization parameter was along the resulting curve. The $\mathbf{L}$-curve will be provided in this paper.

Because of the characteristic of gradient magnetic fields, the shape of $x$-coil is symmetrical related to $z=0$ plane and $x=0$ plane. Rotated the $x$-coil $90^{\circ}$, the shape of $y$-coil is obtained, so only the shielded $x$-coils are considered in this design. $Z$-gradient coil is generally in the form of "Maxwell pair" and which is symmetrical with regard to $z=0$ plane. Based on the symmetry of the coils and the magnetic fields, the design is therefore simplified so as to improve the efficiency.

\section{OUR METHOD}

For the gradient coils of superconducting MRI device, the currents are distributed on a cylindrical surface, so it is natural to use cylindrical coordinates to retain the symmetry of this system. As shown in Fig. 1, the $z$ axis is taken to lie along the axis of this cylinder, radius and length of the primary coil are $R_{p}$ and $L_{p}$, respectively; that of the shielding coil is $R_{s}$ and $L_{s}$, correspondingly. We assume the shielded region's radius is $R_{0}$ and length $L_{0}$, and note the radius of DSV is $r_{D S V}$.

Based on Biot-Savar's law, the magnetic flux density at any point $\left(x_{0}, y_{0}, z_{0}\right)$ in space $\vec{B}\left(x_{0}, y_{0}, z_{0}\right)$, generated by a coil current density, is generally given by

$$
\vec{B}\left(x_{0}, y_{0}, z_{0}\right)=\frac{\mu_{0}}{4 \pi} \iint_{s} \frac{\vec{J} \times \vec{r}}{r^{3}} d s
$$

where $\mu_{0}$ is air permeability, $\vec{J}$ is the current density, $\vec{r}$ is the displacement vector from the source point of the coil to the field point $\left(x_{0}, y_{0}, z_{0}\right)$. There is no current flow in the radial direction in the surface of gradient coil; hence, $\vec{J}$ has only $z$ and $\theta$ components, which are related to each other using current continuity equation $\nabla \cdot \vec{J}=0$.

Based on the symmetry of these gradient coils, the current density can be expanded into Fourier series. For $x$-gradient coils, the current density can be expanded as follows,

$$
\begin{gathered}
J_{\theta}(\theta, z)=\cos (\theta) \sum_{n=1}^{N} a_{n} \cos (2 n \pi z / L) \\
J_{z}(\theta, z)=\sin (\theta) \sum_{n=1}^{N} \frac{a_{n} \cdot L}{2 n \pi R} \sin (2 n \pi z / L)
\end{gathered}
$$

where $a_{n}$ is the unknown Fourier coefficient, $N$ represents the finite term of the Fourier series expansion, $R$ and $L$ are the radius and length of the coil, respectively.

According to (1), (2) and (3), we can get the $z$ component of the magnetic flux density of point $\left(x_{0}, y_{0}, z_{0}\right)$

$$
B_{z}\left(x_{0}, y_{0}, z_{0}\right)=\sum_{n=1}^{N} a_{n} \cdot k_{n}
$$

where

$k_{n}=\frac{\mu_{0} R}{4 \pi} \int_{-\frac{L}{2}}^{\frac{L}{2}} \int_{0}^{2 \pi} \frac{\cos \left(\frac{2 n \pi z}{L}\right)\left(R-y_{0} \sin (\theta)-x_{0} \cos (\theta)\right)}{r^{3}} d \theta d z$

and

$$
r=\sqrt{\left(x_{0}-R \cos (\theta)\right)^{2}+\left(y_{0}-R \sin (\theta)\right)^{2}+\left(z_{0}-z\right)^{2}}
$$

Select $M$ points in the DSV and $Q$ points in the shielded region, $B_{z}$ of these points generated by current densities of primary coil and shielding coil can be obtained

$$
B_{z, i}=\sum_{n=1}^{N_{p}} a_{n}^{p} K_{i, n}^{p}+\sum_{n=1}^{N_{s}} a_{n}^{s} K_{i, n}^{s}, i=1, \cdots, M+Q
$$

where $B_{z, i}$ is the value of $B_{z}$ at the $i$-th constraint point, $N_{p}$ and $N_{s}$ are the number of Fourier coefficients for primary coils and shielding coils, respectively. $a_{n}^{p}$ and $a_{n}^{s}$ are unknown Fourier coefficients for these two coils, respectively. The coefficients $K_{i, n}^{p}$ and $K_{i, n}^{s}$ are the coefficients from the two coils, which can be calculated by substituting the parameters of coils and the location of constraint point into coefficient $k_{n}$.

Given the desired magnetic fields in the DSV and shielded region, we can obtain linear equations in a matrix form by using least-squares minimization

$$
\mathbf{A x}=\mathbf{b}
$$


where $\mathbf{x}=\left\{x_{1}, x_{2}, \cdots x_{N_{p}+N_{s}}\right\}^{\tau}$ is the column vector of Fourier coefficients, $x_{j}=a_{j}^{p}\left(j=1,2, \cdots, N_{p}\right), x_{N_{p}+j}=$ $a_{j}^{s}\left(j=1,2, \cdots, N_{s}\right) ; \mathbf{b}=\left\{b_{1}, b_{2}, \cdots, b_{M+Q}\right\}^{\tau}$ is the column vector of desired $B_{z}$ of the constraint points, $b_{i}=$ $G_{x} \cdot \hat{x}_{i}(i=1,2, \cdots, M), b_{M+i}=0(i=1,2, \cdots, Q)$, $G_{x}$ is the given gradient strength of the $x$-gradient coil, $\hat{x}_{i}$ is the $x$-axis postion of the $i$-th sample point. For the matrix $\mathbf{A}=\left\{\alpha_{i j}\right\}_{(M+Q) \times\left(N_{p}+N_{s}\right)}$, the elements of which are $\alpha_{i j}=K_{i, j}^{p}\left(i=1,2, \cdots, M+Q ; j=1,2, \cdots, N_{p}\right)$; $\alpha_{i\left(N_{p}+j\right)}=K_{i, j}^{s}\left(i=1,2, \cdots, M+Q ; j=1,2, \cdots, N_{s}\right)$.

Equation (8) is ill-conditioned and the little practical value in its present form. A regularization strategy, well-known Tikhonov regularization method, was adopted to obtain Fourier coefficients. The regularization form is

$$
\mathbf{x}_{\lambda}=\underset{\mathbf{x}}{\arg \min }\left\{\|\mathbf{A x}-\mathbf{b}\|_{2}^{2}+\lambda \cdot\|\mathbf{L} \mathbf{x}\|_{2}^{2}\right\}
$$

where $\lambda>0$ is the regularization parameter, $\mathbf{L}$ is constraint matrix deduced from a function $F(\cdot)$.

Assume the penalty function $F(\cdot)$ is defined as

$$
F=\int_{-\frac{L}{2}}^{\frac{L}{2}} \int_{0}^{2 \pi}\left[\left(\frac{1}{R} \cdot \frac{\partial J_{z}}{\partial \theta}\right)^{2}+\left(\frac{\partial J_{\theta}}{\partial z}\right)^{2}\right] R d \theta d z
$$

here the penalty function is used to reduce the maximum current density variation and widen the wire spacing. If we want to get coils with small stored energy or dissipated power, $F(\cdot)$ can be written as the expression of energy or dissipated power.

$\mathbf{L}$ is deduced from the summation of penalty functions of primary coil and shielding coil. For $x$-gradient coils,

$$
\mathbf{L}_{n}=\int_{-\frac{L_{p}}{2}}^{\frac{L_{p}}{2}} \int_{0}^{2 \pi}\left(\frac{L_{p}^{2}}{4 n^{2} \pi^{2} R_{p}^{4}}+\frac{4 n^{2} \pi^{2}}{L_{p}^{2}}\right) \cos ^{2}(\theta) \cdot \sin ^{2}\left(\frac{2 n z \pi}{L_{p}}\right) \cdot R_{p} d \theta d z
$$

where $n=1,2, \cdots, N_{p}$

$$
\mathbf{L}_{n+N_{p}}=\int_{-\frac{L_{s}}{2}}^{\frac{L_{S}}{2}} \int_{0}^{2 \pi}\left(\frac{L_{s}^{2}}{4 n^{2} \pi^{2} R_{s}^{4}}+\frac{4 n^{2} \pi^{2}}{L_{S}^{2}}\right) \cos ^{2}(\theta) \cdot \sin ^{2}\left(\frac{2 n z \pi}{L_{s}}\right) \cdot R_{S} d \theta d z
$$

where $n=1,2, \cdots, N_{s}$.

Then, we can get the errors of magnetic fields in the DSV and shielded region,

$$
\varepsilon=\left(\varepsilon_{i}\right)_{n \times 1}=\mathbf{A x}-\mathbf{b} .
$$

Hence, we have $\varepsilon^{1}=\max \left(\left|\frac{\varepsilon_{i}}{b_{i}}\right|\right) \leq 5 \%, i=1,2, \cdots, M$ and $\varepsilon^{2}=\max \left(\left|\varepsilon_{i}\right|\right) \leq 5.0 \times 10^{-4}, i=M+1, M+2, \cdots, M+$ $Q$.

When the Fourier coefficients are found, the current densities of the primary coil and shielding coil will be obtained. An auxiliary stream function $\vec{S}$ is introduced to realize the required surface current density using a set of discrete wires carrying a constant current. The stream function can be obtained directly from the current density expression analytically based on the following equation

$$
\vec{J}=\nabla \times \vec{S}
$$

Assume the number of current loop is $N_{t}$, then the required current $I$ in the individual wire is given by

$$
I=\frac{S_{\max }-S_{\min }}{N_{t}}
$$

where $S_{\max }$ and $S_{\min }$ are the maximum and minimum values of the stream function. The locations of the wires are given by the contour lines whose values are defined by

$$
S=S_{\min }+\left(i-\frac{1}{2}\right) \cdot I, i=1,2, \cdots, N_{t} .
$$

The magnetic fields are recalculated from the discrete wire lines using the Biot-Savart law. Denote $B_{z c a l, i}$ is the value of $B_{z}$ at the $i$-th point generated by all wire lines, $\varepsilon^{3}=\max \left(\left|\frac{B_{z c a l, i}-b_{i}}{b_{i}}\right|\right), i=1,2, \cdots, M$ and $\varepsilon^{4}=$ $\max \left(\left|B_{z c a l, i}\right|\right), i=M+1, M+2, \cdots, M+Q$. We finish the design when $\varepsilon^{3} \leq 5 \%$ and $\varepsilon^{4} \leq 5.0 \times 10^{-4}$. Otherwise, increase the number of current loop and have another discretization. The corresponding procedure is shown in Algorithm 1.

For $z$-gradient coil, there is only current flow in the angular direction in the coil surface, the current density can be expanded as

$$
J_{\theta}(z)=\sum_{n=1}^{N} a_{n} \sin \left(\frac{2 n \pi}{L} z\right) .
$$

With the similar design as that of $x$-gradient coils, the discrete $z$-gradient coils can be obtained.

\section{RESUlTS AND Discussion}

We have designed cylindrical shielded $x$ - and $z$-gradient coils to test the proposed method. The designed coils are required to produce a linear magnetic field strength of $G_{x}=$ $\frac{\partial B_{z}}{\partial x}=30 \mathrm{mT} / \mathrm{m}$ and $G_{z}=\frac{\partial B_{z}}{\partial z}=30 \mathrm{mT} / \mathrm{m}$ in a $0.45 \mathrm{~m} \mathrm{DSV}$ size. The geometry parameters and requirements are specified in Table I.

In the design, the appropriate $\lambda$ value was determined by the $\mathbf{L}$-curve of regularization tool. The $\mathbf{L}$-curve plots for $x$-and $z$-gradient coils are shown in Fig. 2. Usually, the smaller the value of $\lambda$, the better the linearity of gradient field, and the larger the value of the stream function. There is a balance between gradient linearity and stream function. The value of $\lambda$ is set as that of the inflexion point in the L-curve, $1.8117 \times 10^{-10}$ and $8.4731 \times 10^{-7}$ for $x$ - and $z$-gradient coils, respectively.

Fig. 3 (a)/(b) and (c)/(d) illustrate the three-dimensional patterns of shielded $x$ - and $z$-coils separately. We see that the current of each coil is asymmetrical about the $x y$-plane; the current direction of the primary coil is opposite to that of the shielding coil. Especially, the current is asymmetrical about the $y z$-plane for $x$-coils.

Fig. 4 (a)/(b) and (c)/(d) illustrates the planar discrete patterns of shielded gradient coils separately. There is one quadrant for $x$-coils and other three quadrants can be acquired in terms of the symmetry of the current distribution. 


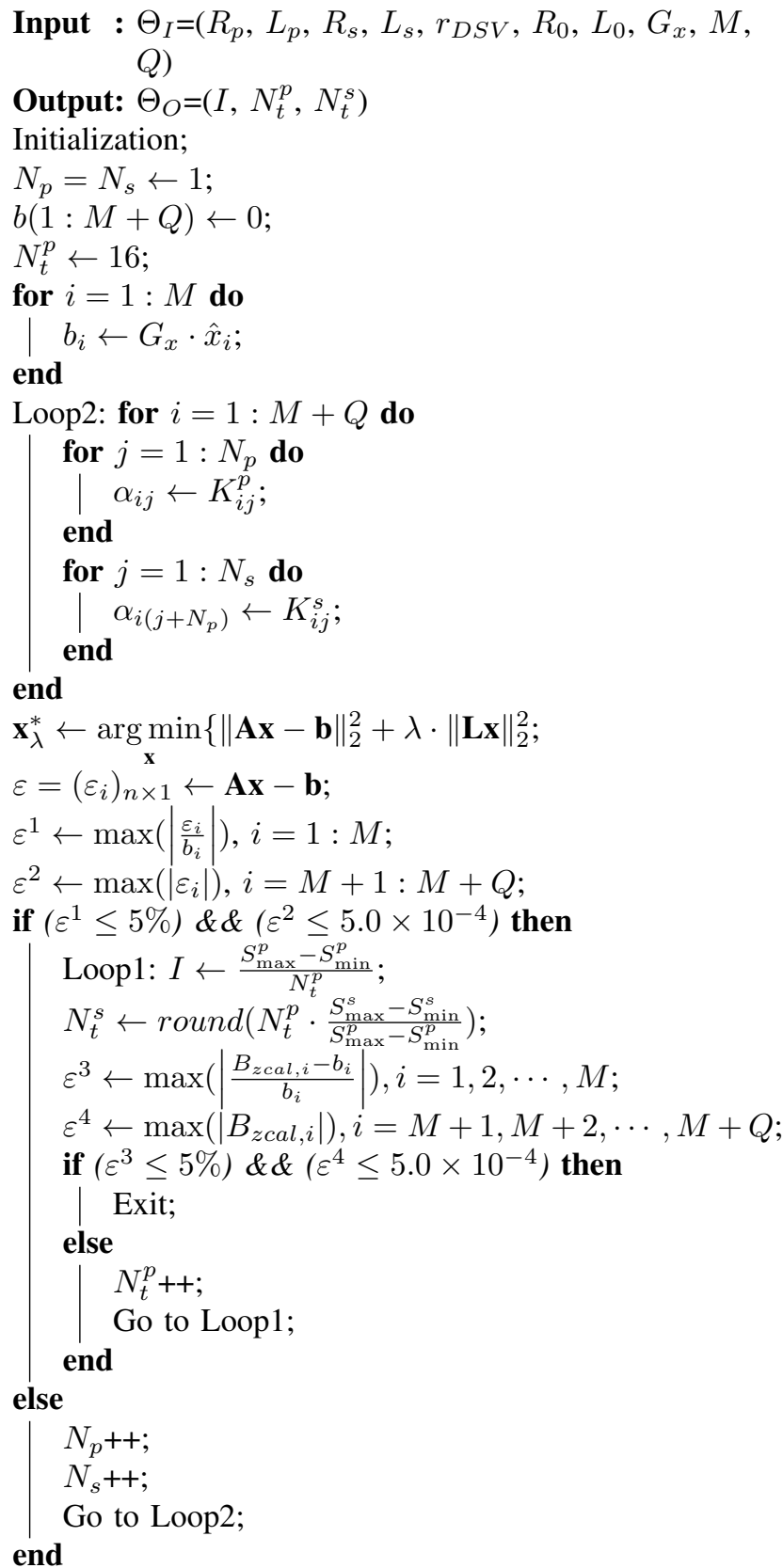

Algorithm 1: The algorithm designed

TABLE I

CHARACTERISTICS OF THE SHIELDED X-AND Z- GRADIENT COILS DESIGNS

\begin{tabular}{|c|c|c|}
\hline Item & x-coils & z-coils \\
\hline Coil length(P/S) $(\mathrm{m})$ & $1.38 / 1.48$ & $1.38 / 1.48$ \\
\hline Coil radius(P/S) $(\mathrm{m})$ & $0.32 / 0.37$ & $0.34 / 0.40$ \\
\hline DSV (diameter) (m) & 0.45 & 0.45 \\
\hline Gradient strength (mT/m) & 30 & 30 \\
\hline Number of turns (P/S) & $38 / 26$ & $36 / 25$ \\
\hline Wire current (A) & 477.45 & 427.98 \\
\hline
\end{tabular}
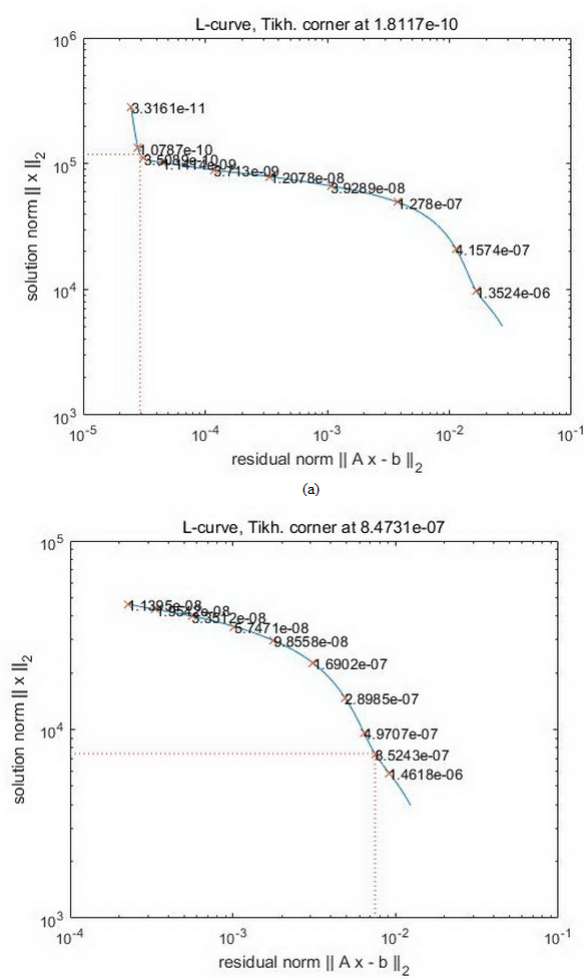

Fig. 2. The $L$-curve plots for the Tikhonov regularization of (a) $x$-gradient coils and (b) $z$-gradient coils. (a)

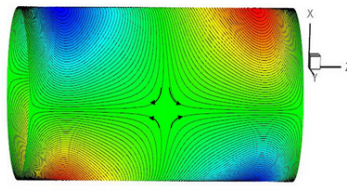

(c)

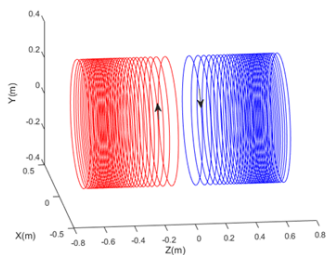

(b)

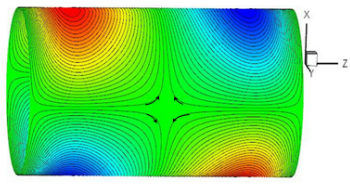

(d)

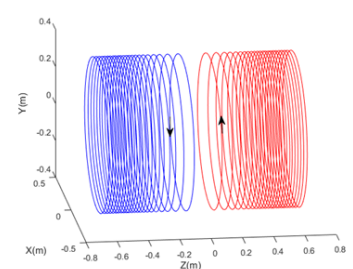

Fig. 3. The demonstration of three-dimensional shielded coils (a) primary $x$-coil, (b) shielding $x$-coil, (c) primary $z$-coil, (d) shielding $z$-coil.

A gradient power supply device is shared by the primary and the shielding coils; there is the same current supplied for shielded coils. In order to get the desired magnetic field distribution, the turns of current loop for shielded coils should be balanced.

To validate the results of the numerical methodology proposed in this paper, the magnetic field is calculated from the wire patterns shown in Fig. 4. The nonlinearities of the gradient field in the DSV and magnetic field distributions in the shielded region, generated by using shielded coils, are shown in Fig. 5. It is seen from Fig. 5 (a)/(b) that the calculated 
(a)

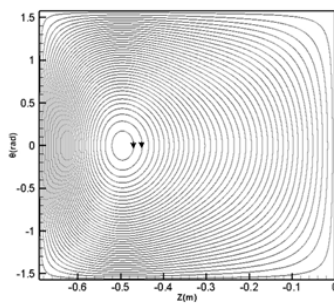

(c)

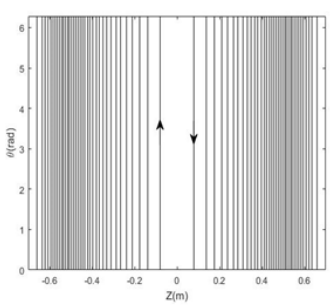

(b)

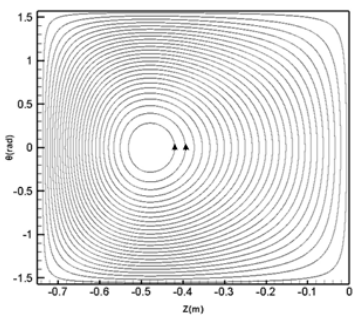

(d)

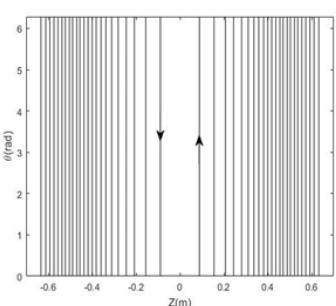

Fig. 4. The planar discrete patterns of shielded coils (a) primary $x$-coil(one quadrant), (b) shielding $x$-coil(one quadrant), (c) primary $z$-coil (whole), and (d) shielding $z$-coil (whole).

(a)

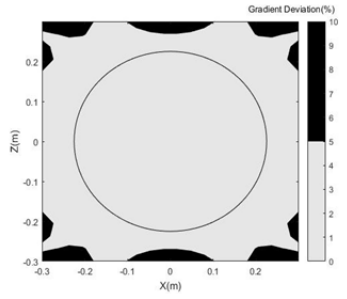

(c)

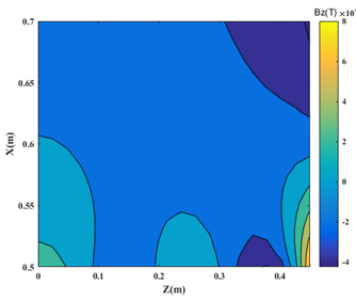

(b)

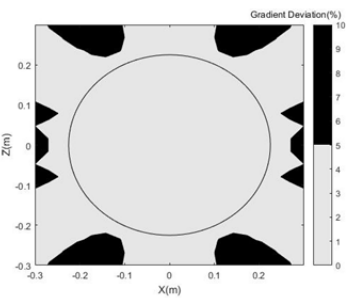

(d)

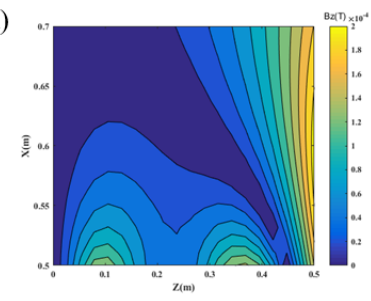

Fig. 5. Gradient field nonlinearity profile in the coronal plane (with 5\% deviation) produced by using (a) $x$-coils and (b) $z$-coils. The magnetic field in the shielded region for the designed (c) $x$-coils and (d) $z$-coils.

gradient fields exactly match with the requirements of our design. Fig. 5 (c)/(d) indicate that the magnetic flux density inside the shielded region is less than 5 Gauss.

For the design of unshielded gradient coils, we can only consider the current density of the primary coil, and select sample points in the DSV.

\section{CONClusion}

The proposed approach for cylinder gradient coils has been formulated and presented. The current densities of the coil surfaces are expanded into Fourier series, the problem is simplified into the solution of Fourier coefficients instead of current densities. The large number of expansion items can improve the accuracy of the design, but also lead to an increase in the complexity of the windings. Actually, the numbers of

Fourier expansion items for shielded coils are adjusted to make the patterns of coils practically implementable. The penalty function mentioned in the design can also be other quantity, such as stored energy and dissipated power etc. to get the coils with small inductance or resistance. The method in this paper can also be used to the design and optimization of other gradient coils.

\section{REFERENCES}

[1] J. Jin, "Electromagnetic analysis and design in magnetic resonance imaging," Informa Healthcare, 1998.

[2] P. Mansfield, and B. Chapman, "Active magnetic screening of coils for static and time-dependent magnetic field generation in NMR imaging," Journal of Physics (E) Scientific Instruments, vol. 19, no. 7, pp. 540545, 1986.

[3] R. Turner, "Minimum inductance coils," Journal of Physics (E) Scientific Instruments, vol. 21, no. 10, pp. 948-952, 1988.

[4] R. Turner, "A target field approach to optimal coil design," Journal of Physics (D) Applied Physics, vol. 19, no. 8, pp. 147- 151, 1986.

[5] R. Turner, and R. M. Bowley, "Passive screening of switched magnetic field gradients," Journal of Physics (E) Scientific Instruments, vol. 19, no. 10 , pp. 876-879, 1986.

[6] R. Turner, "Gradient coil design: A review of methods," Magnetic Resonance Imaging, vol. 11, no. 7, pp. 903-920, 1993.

[7] K. Yoda, "Analytical design method of selfshielded planar coils," Journal of Applied Physics, vol. 67, no. 9, pp. 4349-4353, 1990.

[8] D. Tomasi, "Optimization of biplanar gradient coils for magnetic resonance imaging," Brazilian Journal of Physics, vol. 36, no. 1A, pp. 23-27, 2006.

[9] J. Leggett, S. Crozier, S. Blackband, B. Beck, and R. W. Bowtell, "Multilayer transverse gradient coil design," Concepts in Magnetic Resonance Part B Magnetic Resonance Engineering, vol. 16B(1), pp. 38-46, 2003.

[10] L. S. Petropoulos and M. A. Morich, "Novel gradient coil set with an interstitial gap for interventional nuclear magnetic resonance applications," IEEE Transactions on Magnetics, vol. 33, no. 5, pp. 4107-4109, 1997.

[11] S. Shvartsman, M. Morich, G. Demeester, and Z. Zhai, "Ultrashort shielded gradient coil design with 3D geometry," Concepts in Magnetic Resonance Part B Magnetic Resonance Engineering, vol. 26B(1), pp. $1-15,2005$.

[12] L. S. Petropoulos, "Finite size disc gradient coil set for open vertical field magnets," Magnetic Resonance Imaging, vol. 18, no. 5, pp. 615624, 2000.

[13] L. K. Forbes, M. A. Brideson, and S. Crozier, "A target-field method to design circular biplanar coils for asymmetric shim and gradient fields," IEEE Transactions on Magnetics, vol. 41, no. 6, pp. 2134-2144, 2005.

[14] Y. Wang, X. Xin, F. Liu, and S. Crozier,"Spiral gradient coil design for use in cylindrical MRI systems," IEEE Transactions on Biomedical Engineering, vol. 65, no. 4, pp. 911-920, 2018.

[15] Y. Wang, F. Liu, X. Zhou, and S. Crozier,"Design of transverse head gradient coils using a layer-sharing scheme," Journal of Magnetic Resonance, vol. 278, pp. 88-95, 2017.

[16] G. Shou, L. Xia, F. Liu, M. Zhu, Y. Li, and S. Crozier,"MRI coil design using boundary-element method with regularization technique: a numerical calculation study," IEEE Transactions on Magnetics, vol. 46, no. 4, pp. 1052-1059, 2010.

[17] R. A. Lemdiasov and R. Ludwig, "A stream function method for gradient coil design," Concepts in Magnetic Resonance Part B Magnetic Resonance Engineering, vol. 26B(1), pp. 67-80, 2010.

[18] P. C. Hansen, "Regularization Tools: A Matlab package for analysis and solution of discrete ill-posed problems," Numerical Algorithms, vol. 6, no. 1, pp. 1-35, 1994. 